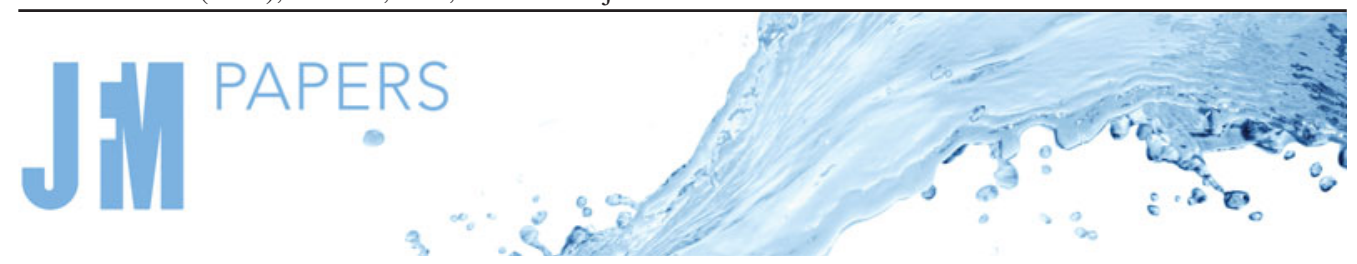

\title{
Boussinesq and non-Boussinesq gravity currents propagating on unbounded uniform slopes in the deceleration phase
}

\author{
Albert Dai ${ }^{1} \uparrow$ and Yu-Lin Huang ${ }^{1}$ \\ ${ }^{1}$ Department of Engineering Science and Ocean Engineering, National Taiwan University, Taipei, Taiwan
}

(Received 13 October 2020; revised 15 March 2021; accepted 29 March 2021)

Boussinesq and non-Boussinesq gravity currents produced from a finite volume of heavy fluid propagating into an environment of light ambient fluid on unbounded uniform slopes in the range $0^{\circ} \leq \theta \leq 12^{\circ}$ are reported. The relative density difference $\epsilon=$ $\left(\rho_{1}-\rho_{0}\right) / \rho_{0}$ is varied in the range $0.05 \leq \epsilon \leq 0.15$ in this study, where $\rho_{1}$ and $\rho_{0}$ are the densities of the heavy and light ambient fluids, respectively. Our focus is on the influence of the relative density difference on the deceleration phase of the propagation. In the early deceleration phase, the front location history follows the power relationship $\left(x_{f}+x_{0}\right)^{2}=\left(K_{I} B\right)^{1 / 2}\left(t+t_{I}\right)$, where $\left(x_{f}+x_{0}\right)$ is the front location measured from the virtual origin, $K_{I}$ an experimental constant, $B$ the total buoyancy, $t$ the time and $t_{I}$ the $t$ intercept. The dimensionless constant $K_{I}$ is influenced by the slope angle and the relative density difference. In the late deceleration phase for the gravity currents on the steeper slopes in this study $\left(12^{\circ}, 9^{\circ}\right.$ and $\left.6^{\circ}\right)$, an 'active' head separates from the body of the current and the front location history follows the power relationship $\left(x_{f}+x_{0}\right)^{8 / 3}=$ $K_{V S} B^{2 / 3} V_{0}^{2 / 9} v^{-1 / 3}\left(t+t_{V S}\right)$, where $K_{V S}$ is an experimental constant, $V_{0}$ the total volume of heavy fluid, $v$ the kinematic viscosity of fluid and $t_{V S}$ the $t$ intercept. The dimensionless constant $K_{V S}$ is shown to be influenced by the slope angle but not significantly influenced by the relative density difference. In the late deceleration phase for the gravity currents on the milder slopes in this study $\left(3^{\circ}\right.$ and $\left.0^{\circ}\right)$, the gravity currents maintain an integrated shape without violent mixing with the ambient fluid and the front location history follows the power relationship $\left(x_{f}+x_{0}\right)^{4}=K_{V M} B^{2 / 3} V_{0}^{2 / 3} v^{-1 / 3}\left(t+t_{V M}\right)$, where $K_{V M}$ is an experimental constant and $t_{V M}$ the $t$ intercept. The dimensionless constant $K_{V M}$ is shown to be influenced by both the slope angle and the relative density difference. While the influence of the relative density difference on $K_{V M}$ is carried along for the gravity currents on the milder slopes in the late deceleration phase, the relative density difference

$\dagger$ Email address for correspondence: hdai@ntu.edu.tw

(C) The Author(s), 2021. Published by Cambridge University Press. This is an Open Access article, distributed under the terms of the Creative Commons Attribution licence (http://creativecommons.org/ licenses/by/4.0/), which permits unrestricted re-use, distribution, and reproduction in any medium, provided the original work is properly cited. 


\section{A. Dai and Y.-L. Huang}

interestingly has no significant influence on $K_{V S}$ for the gravity currents on the steeper slopes in the late deceleration phase. Our results suggest that the non-Boussinesq gravity currents on the milder slopes may remain non-Boussinesq ones in the late deceleration phase while the non-Boussinesq gravity currents on the steeper slopes may have become Boussinesq ones in the late deceleration phase.

Key words: gravity currents

\section{Introduction}

Gravity currents are flows driven by density differences and occur ubiquitously in natural and man-made environments (Simpson 1997; Ungarish 2009). For a long time, the lock-exchange set-up has served as a paradigm configuration for studying the propagation of gravity currents (Shin, Dalziel \& Linden 2004; Adduce, Sciortino \& Proietti 2012; La Rocca et al. 2012a,b; Ottolenghi et al. 2016a). In the classic lock-exchange experiments, two fluids of different densities are separated by a removable lock gate in a long horizontal channel. The motion of the two fluids in the channel is initiated when the lock gate is removed. The gravity currents in the classic lock-exchange set-up are also called planar gravity currents as the flows are bounded in the spanwise direction by the channel walls.

Gravity currents may also be influenced by the presence of a sloping boundary. In the literature, a number of studies have investigated planar gravity currents propagating on a favourable slope (Beghin, Hopfinger \& Britter 1981; Bonnecaze \& Lister 1999; Pawlak \& Armi 2000; Rastello \& Hopfinger 2004; Maxworthy \& Nokes 2007; Maxworthy 2010; Dai 2013a, 2014, 2015; Dai \& Huang 2016; Negretti, Flòr \& Hopfinger 2017; Ottolenghi, Cenedese \& Adduce 2017b; Steenhauer, Tokyay \& Constantinescu 2017), while some have investigated planar gravity currents propagating on an adverse slope (Jones et al. 2014; Marleau, Flynn \& Sutherland 2014; Lombardi et al. 2015; Ottolenghi et al. 2016b, 2017a). For the planar gravity currents propagating on a favourable slope, Beghin et al. (1981) observed that planar gravity currents may go through an acceleration phase followed by a deceleration phase, and developed the thermal theory to describe the two phases of motion. Dai $(2013 a, 2014)$ further categorised the deceleration phase of the planar gravity currents propagating on a favourable slope into an early stage, where the buoyancy force is in balance with the inertia force, and a late stage, where the buoyancy force is in balance with the viscous force.

Compared with the planar gravity currents, the gravity currents propagating on a horizontal or sloping boundary without being bounded in the spanwise direction have received less attention (Cantero, Balachandar \& Garcia 2007; La Rocca et al. 2008; Sahuri et al. 2015; Dai \& Wu 2016; Inghilesi et al. 2018). However, this configuration is more similar to that of turbidity currents down a continental shelf and powder snow avalanches (Hopfinger 1983; Ouillon, Meiburg \& Sutherland 2019). The gravity currents propagating on unbounded uniform slopes may also go through an acceleration phase followed by a deceleration phase. Using the shallow water model, Webber, Jones \& Martin (1993) and Tickle (1996) predicted that the gravity currents on unbounded uniform slopes would take a self-similar circular wedge shape. Using laboratory experiments, Ross, Linden \& Dalziel (2002) showed that the Boussinesq gravity currents on unbounded uniform slopes take a shape which is more akin to a triangular wedge. Using high-resolution direct numerical simulations, Zgheib, Ooi \& Balachandar (2016) confirmed the observations made by Ross et al. (2002) and reported that, for the Boussinesq gravity currents propagating on 


\section{Gravity currents on unbounded uniform slopes}

unbounded uniform slopes, the heavy fluid may initially propagate outward from the lock in a diverging manner but converge towards the centre of the gravity currents at a later time.

The Boussinesq gravity currents propagating on unbounded uniform slopes have recently been investigated by Dai \& Huang (2020). It is reported that there are two stages of the deceleration phase for the Boussinesq gravity currents on unbounded uniform slopes and different power relationships apply in the early and late stages of the deceleration phase. This study is a continuation of the investigation on the Boussinesq gravity currents on unbounded uniform slopes conducted by the authors. Our focus is on the influence of the relative density difference on the deceleration phase of the propagation. The relative density difference between the heavy fluid and light ambient fluid $\epsilon=\left(\rho_{1}-\rho_{0}\right) / \rho_{0}$ is varied in the range $0.05 \leq \epsilon \leq 0.15$ in this study, where $\rho_{1}$ and $\rho_{0}$ are the densities of the heavy and light ambient fluids, respectively. We may quantitatively measure the influence of the slope angle and the relative density difference on the propagation of gravity currents. As we will show, the non-Boussinesq gravity currents on the steeper unbounded uniform slopes in this study $\left(12^{\circ}, 9^{\circ}\right.$ and $\left.6^{\circ}\right)$ may have become Boussinesq ones in the late deceleration phase while the non-Boussinesq gravity currents on the milder unbounded uniform slopes in this study $\left(3^{\circ}\right.$ and $\left.0^{\circ}\right)$ may remain non-Boussinesq ones in the late deceleration phase. In $\S 2$, we summarise the theoretical relationships between the front location and time in the early stage and late stage of the deceleration phase. The experimental set-up is described in $\S 3$. Qualitative and quantitative results are presented in $\S 4$ and the conclusions are drawn in $\S 5$.

\section{Theoretical background}

The configuration of the problem is sketched in figure 1. The density of the heavy fluid in the lock is $\rho_{1}$ and the density of the light ambient fluid is $\rho_{0}$. The relative density difference is $\epsilon=\left(\rho_{1}-\rho_{0}\right) / \rho_{0}$ or equivalently we may define the density ratio between the ambient and heavy fluids as $\gamma=\rho_{0} / \rho_{1}$. The density ratio is related to the relative density difference via $\gamma=(1+\epsilon)^{-1}$. For the non-Boussinesq case, here we generalise the Boussinesq wedge integral model in Dai \& Huang (2020) without invoking the Boussinesq approximation. In the wedge integral model, the width and height of the wedge are taken as $b=\pi l$ and $h=l \tan \theta$, where $l$ represents the length of the wedge. The volume of the wedge is $V=S_{3} l^{3} \tan \theta$, where $S_{3}=1 / 3$ is a shape factor for a triangular wedge (Ross et al. 2002).

The convection of the gravity current is driven by the density difference between the heavy fluid in the current and the light ambient fluid in the environment. The influence of the drag force is relatively small compared with the influence of turbulent entrainment, as discussed by Dai $(2013 a, b, 2014)$. Therefore, the drag force is neglected in our wedge integral model for the non-Boussinesq case. Without the Boussinesq approximation, the linear momentum equation takes the form

$$
\frac{\mathrm{d}}{\mathrm{d} t}\left(\left(\rho+\rho_{0} C_{A}\right) V U\right)=\rho_{0} B \sin \theta,
$$

where $\rho$ is the density of the heavy fluid in the current, $C_{A}$ is the 'added mass' coefficient, which takes into account the ambient fluid carried along with the gravity currents, $U$ is the velocity of the centre of mass of the current wedge, $B=V_{0} g\left(\rho_{1}-\rho_{0}\right) / \rho_{0}$ is the buoyancy which is conserved during the propagation of gravity currents and $t$ is the time. The density of the heavy fluid in the current, $\rho$, is not a constant and will gradually decrease due to entrainment of light ambient fluid as the current propagates downslope. The added 


\section{A. Dai and Y.-L. Huang}

(a)

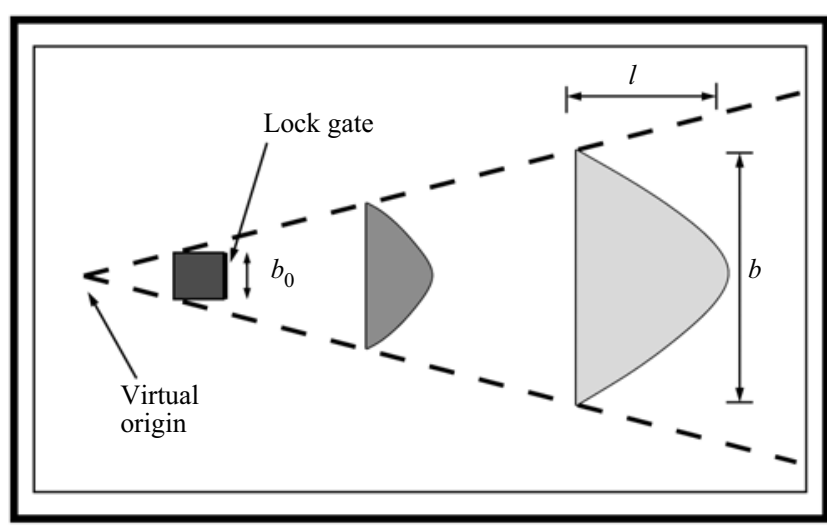

(b)

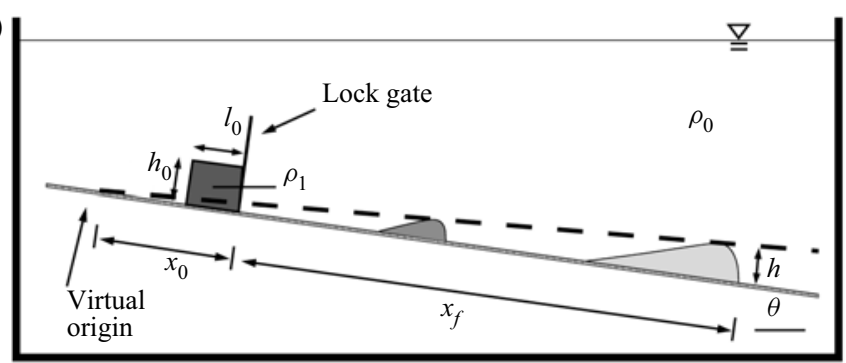

Figure 1. Sketch of the experimental set-up. The uniform slope makes an angle $\theta$ with the horizontal plane. Panel $(a)$ shows the top view and panel $(b)$ shows the side view of the tank. In panel $(a), l$ and $b$ represent the length and width of the self-similar wedge. In panel $(b), h$ represents the height of the wedge. The heavy fluid initially contained in the lock has density $\rho_{1}$ while the light ambient fluid has density $\rho_{0}$. The front location $x_{f}$ is measured from the lock gate and the virtual origin is at a distance $x_{0}$ upslope of the lock gate.

mass coefficients for a circular cylinder and a sphere are given as 1 and 0.5 , respectively, by Batchelor (1967). For a streamlined wedge of width $b$ and length $l$, the added mass coefficient for the wedge is estimated as $C_{A}=0.5$ in this study (Korotkin 2008).

With the assumption of turbulent entrainment (Ellison \& Turner 1959), the mass conservation takes the form

$$
\frac{\mathrm{d} V}{\mathrm{~d} t}=\alpha U A_{E}
$$

where $A_{E}=S_{4} l^{2} \tan \theta$ is the area over which the entrainment takes place, $S_{4}=2 \sqrt{2}$ is another shape factor (Ross et al. 2002) and $\alpha$ is the entrainment coefficient. From (2.2) and using $U=\mathrm{d} x / \mathrm{d} t$, we may derive

$$
l=\frac{S_{4} \alpha}{3 S_{3}} x
$$

where $x$ is the distance measured from the 'virtual origin' to the centre of mass of the wedge. By using $U=\mathrm{d} x / \mathrm{d} t$ and the chain rule $\mathrm{d} / \mathrm{d} t=U \mathrm{~d} / \mathrm{d} x$ to solve (2.2), the independent variable in (2.2) is transformed from $t$ to $x$. The 'virtual origin' is located $x_{0}$ upslope of the lock gate and can be identified by extrapolating the width of the wedge in the upslope direction, as shown in figure 1. Upon substituting (2.3) into (2.1), we may 


\section{Gravity currents on unbounded uniform slopes}

derive the solution for the momentum equation, i.e.

$$
U^{2}=U_{0}^{2} \frac{(1+\Delta)^{2}}{\left(X^{3}+\Delta\right)^{2}}+U_{\infty}^{2} \frac{\left(X^{4}-1\right)+4 \Delta(X-1)}{\left(X^{3}+\Delta\right)^{2}},
$$

where $U_{0}$ is the initial mass-centre velocity,

$$
U_{\infty}^{2}=\frac{27 B S_{3}^{2} \cos \theta}{2 x_{0}^{2} \alpha^{3} S_{4}^{3}\left(1+C_{A}\right)} \quad \text { and } \quad \Delta=\frac{1-\gamma}{\gamma} \frac{27 S_{3}^{2} V_{0}}{x_{0}^{3} \alpha^{3} S_{4}^{3} \tan \theta\left(1+C_{A}\right)}
$$

and $X=x / x_{0}$ is introduced for a clear and concise form of the solution (2.4).

Our wedge integral model predicts that, for gravity currents starting from a quiescent condition, the centre of mass velocity reaches its maximum at $X=X_{M}$, which satisfies the following relationship

$$
X_{M}^{6}+8 \Delta X_{M}^{3}-3(4 \Delta+1) X_{M}^{2}+2 \Delta^{2}=0,
$$

by setting the spatial derivative of (2.4) to zero. In the Boussinesq limit when $\epsilon \rightarrow 0$ (i.e. $\gamma \rightarrow 1$ and $\Delta \rightarrow 0$ ), we note that $X_{M} \rightarrow 3^{1 / 4}$ as previously shown by Dai \& Huang (2020) in the Boussinesq case.

Since in the experiments the front location is more easily measurable than the centre of mass, we use the geometric relation for a triangular wedge $\left(x_{f}+x_{0}\right)=x+l / 2$, i.e. $\left(x_{f}+x_{0}\right)=\left(1+S_{4} \alpha / 6 S_{3}\right) x$, to express the front location $x_{f}$ in place of the centre of mass location. Please note that the front location $x_{f}$ is measured from the lock gate and the distance from the virtual origin to the front is given by $\left(x_{f}+x_{0}\right)$. Consequently, the front velocity is related to the centre of mass velocity via $U_{f}=\left(1+S_{4} \alpha / 6 S_{3}\right) U$ and the maximum front velocity $U_{f_{\max }}$ can be expressed as

$$
U_{f_{\text {max }}}=\left(1+\frac{S_{4} \alpha}{6 S_{3}}\right) \sqrt{\frac{27 B S_{3}^{2} \cos \theta}{2 x_{0}^{2} \alpha^{3} S_{4}^{3}\left(1+C_{A}\right)}} \sqrt{\frac{\left(X_{M}^{4}-1\right)+4 \Delta\left(X_{M}-1\right)}{\left(X_{M}^{3}+\Delta\right)^{2}}} .
$$

For gravity currents sufficiently far into the deceleration phase such that $X \gg 1$ and $X \gg 4 \Delta$, the front velocity approaches the asymptote

$$
U_{f}=\left(1+\frac{S_{4} \alpha}{6 S_{3}}\right)^{2} \sqrt{\frac{27 B S_{3}^{2} \cos \theta}{2 \alpha^{3} S_{4}^{3}\left(1+C_{A}\right)}}\left(x_{f}+x_{0}\right)^{-1} .
$$

The wedge integral model has been obtained with the hypothesis of non-trivial or moderate values of relative density difference and will be applied for relative density differences in the range $0.05 \leq \epsilon \leq 0.15$. The application to cases with even larger relative density difference at $\epsilon>0.15$ is not corroborated and is beyond the scope of the present work. Since $\Delta \approx O(\epsilon)$, as we considered the relative density difference in the range $0.05 \leq \epsilon \leq$ 0.15 , the condition $X \gg 4 \Delta$ is no more stringent than $X \gg 1$. Upon integration, (2.8) can be rewritten in the following form with an integration constant $t_{I}$

$$
\left(x_{f}+x_{0}\right)^{2}=\left(K_{I} B\right)^{1 / 2}\left(t+t_{I}\right),
$$

where

$$
K_{I}=\left(1+\frac{S_{4} \alpha}{6 S_{3}}\right)^{4} \frac{54 S_{3}^{2} \cos \theta}{\alpha^{3} S_{4}^{3}\left(1+C_{A}\right)},
$$

which is independent of $B$ and is a function of the slope angle $\theta$. According to the Buckingham theorem, the dimensionless constant $K_{I}$ should be a function of the slope 


\section{A. Dai and Y.-L. Huang}

angle and the relative density difference. The dependence of the dimensionless constant $K_{I}$ on the relative density difference is not explicit in (2.10) but is implicit via the influence of the relative density difference on the entrainment coefficient $\alpha$. The density difference between the heavy fluid in the current and the light ambient fluid stabilises the interface and the entrainment coefficient is expected to decrease as the relative density difference increases (Ellison \& Turner 1959; Hacker, Linden \& Dalziel 1996; Hallworth et al. 1996; Johnson \& Hogg 2013).

From the scaling analysis, it has been shown that the relationship (2.9) is essentially a statement of balance between the buoyancy force and inertia force (Dai \& Huang 2020). In the Boussinesq case, the relative density difference does not play a role since $\epsilon \rightarrow 0$ $(\gamma \rightarrow 1)$. In the non-Boussinesq case, the dimensionless constant $K_{I}$ is a function of both the slope angle and the relative density difference. In this study, we shall term the time period during which (2.9) applies the early stage of deceleration phase.

As will be shown later, for the gravity currents propagating on unbounded uniform slopes equal to or greater than $6^{\circ}$, an 'active' head separates from the body of the current in the late deceleration phase. In the late deceleration phase, when the viscous force becomes more important, the viscous force per unit mass scales as $v\left(x_{f}+x_{0}\right) t^{-1} \delta^{-1} V_{0}{ }^{-1 / 3}$, where the viscous stress per unit density is estimated as $v\left(x_{f}+x_{0}\right) t^{-1} \delta^{-1}$, the thickness of the boundary layer at the interface between the current and ambient fluid is estimated as $\delta \sim(v t)^{1 / 2}$ and $V_{0}{ }^{1 / 3}$ is an estimate for the length scale for the 'active' current wedge (Dai \& Huang 2020). When a balance between the buoyancy force per unit mass, which scales as $B\left(x_{f}+x_{0}\right)^{-3}$, and the viscous force per unit mass, which scales as $v\left(x_{f}+x_{0}\right) t^{-1} \delta^{-1} V_{0}{ }^{-1 / 3}$, is struck for the 'active' current head, the following relationship applies in the late deceleration phase, i.e.

$$
\left(x_{f}+x_{0}\right)^{8 / 3}=K_{V S}\left(\frac{B^{2} V_{0}^{2 / 3}}{v}\right)^{1 / 3}\left(t+t_{V S}\right),
$$

where $K_{V S}$ is an experimental constant and $t_{V S}$ the $t$ intercept.

For the gravity currents propagating on unbounded uniform slopes equal to or less than $3^{\circ}$, the gravity currents maintain an integrated shape. In the early stage of deceleration phase, when the buoyancy and inertia forces are in balance, the front location history similarly follows the relationship (2.9). In the late stage of the deceleration phase, the gravity currents maintain an integrated shape and the viscous stress per unit density, $v\left(x_{f}+x_{0}\right) t^{-1} \delta^{-1}$, is applied over the whole top area, which scales as $\left(x_{f}+x_{0}\right)^{2}$. Therefore, the viscous force per unit mass scales as $v\left(x_{f}+x_{0}\right)^{3} t^{-1} \delta^{-1} V_{0}{ }^{-1}$. When the buoyancy force per unit mass, $B\left(x_{f}+x_{0}\right)^{-3}$, and the viscous force per unit mass are in balance, the front location history follows the power relationship

$$
\left(x_{f}+x_{0}\right)^{4}=K_{V M}\left(\frac{B^{2} V_{0}^{2}}{v}\right)^{1 / 3}\left(t+t_{V M}\right),
$$

where $K_{V M}$ is an experimental constant and $t_{V M}$ the $t$ intercept. The experimental constant $K_{I}$ for the Boussinesq gravity currents propagating on an unbounded horizontal plane can be estimated as 5.71 and 3.62, based on the experiments of Hoult (1972) and Huppert \& Simpson (1980), respectively. The experimental constant $K_{V M}$ for the Boussinesq gravity currents propagating on an unbounded horizontal plane can be estimated as 1.24 based on the experiments of Hoult (1972). In the non-Boussinesq case, according to 


\section{Gravity currents on unbounded uniform slopes}

the Buckingham theorem, the experimental constants $K_{V S}$ and $K_{V M}$ are expected to be functions of both the slope angle and the relative density difference.

\section{Experimental set-up}

A sketch of the tank used in the experiments is provided in figure 1 with top and side views. The tank was used for the Boussinesq gravity currents on unbounded uniform slopes in Dai \& Huang (2020), to which the readers are referred for other details of the experimental set-up.

The rectangular tank has dimensions of $1.6 \mathrm{~m}$ in width, $0.6 \mathrm{~m}$ in depth and $2.5 \mathrm{~m}$ in length and all four sides were constructed by transparent Perspex walls for visualisation purposes. A Perspex board was installed near the bottom of the tank to act as an unbounded uniform slope and the slope angle could be adjusted in the range of $0^{\circ} \leq \theta \leq 12^{\circ}$. The lock has dimensions of $b_{0}=10 \mathrm{~cm}$ in width, $h_{0}=8 \mathrm{~cm}$ in height and $l_{0}=10 \mathrm{~cm}$ in length and was mounted on the upslope end of the Perspex board. The heavy fluid in the lock was set into motion when the gate in the lock was removed.

Potassium permanganate was added in the heavy fluid for visualisation purposes. A Sony HDR-PJ670 was positioned $4 \mathrm{~m}$ above the free surface of ambient fluid for the top view images and a Canon EOS 700D was positioned at approximately $4 \mathrm{~m}$ away from the sidewall of the tank for the side view images. Both cameras have spatial and temporal resolutions of $1920 \times 1080$ at 24 frames per second. The Canon EOS 700D camera was rotated at the same angle as the slope such that the $x$ and $y$ axes in the images align with the downslope and wall-normal directions.

In the experiments, sodium chloride solution was chosen as the heavy fluid while tap water was chosen as the ambient fluid. The kinematic viscosity of the sodium chloride solution is taken to be the same as the tap water as $v=1.1 \times 10^{-2} \mathrm{~cm}^{2} \mathrm{~s}^{-1}$. Densities of the heavy fluid and ambient fluid were measured by a density meter with an accuracy of $10^{-3} \mathrm{~g} \mathrm{~cm}^{-3}$. The relative density differences were chosen at $\epsilon=0.15,0.10$ and 0.05 in the experiments and the reduced gravity values $g_{0}^{\prime}=g\left(\rho_{1}-\rho_{0}\right) / \rho_{0}$ at the three relative density differences were approximately $g_{0}^{\prime} \approx 147.15,98.1$ and $49.05 \mathrm{~cm} \mathrm{~s}^{-2}$, respectively.

The Reynolds numbers at the three relative density differences were $R e=\sqrt{g_{0}^{\prime} h_{0}} h_{0} / v \approx$ 25000,20000 and 15000 , respectively.

\section{Results}

In the following, we shall present the results for the non-Boussinesq gravity currents propagating on unbounded uniform slopes $\theta=12^{\circ}, 9^{\circ}, 6^{\circ}, 3^{\circ}, 0^{\circ}$ in order. The dimensions of the lock and the densities of the heavy fluid and ambient fluid were maintained unchanged throughout the experiments. On each slope angle, at least five repeated runs were performed in order to make qualitative and quantitative observations. Other experimental parameters are listed in table 1.

\subsection{Non-Boussinesq gravity currents on an unbounded uniform $12^{\circ}$ slope}

\subsubsection{Qualitative features}

Figures 2 and 3 show the top view and side view images for a gravity current with $\epsilon=$ $0.15(\gamma=0.87)$ propagating on an unbounded uniform $12^{\circ}$ slope. After the lock gate is removed, the heavy fluid in the lock collapses and spreads outward from the lock. As will be shown later, the maximum front velocity is reached at $t \approx 1.67 \mathrm{~s}$, after which the 


$\begin{array}{lccccc}\text { Case } \theta \text { (deg.) } & \gamma ; \epsilon & g_{0}^{\prime}\left(\mathrm{cm} \mathrm{s}^{-2}\right) & t_{\max }(\mathrm{s}) & x_{f_{\max }}(\mathrm{cm}) & U_{f_{\max }}\left(\mathrm{cm} \mathrm{s}^{-1}\right) \\ \text { 12I } & 0.87 ; 0.15 & 147.18_{-0.02}^{+0.08} & 1.87_{-0.19}^{+0.80} & 32.47_{-2.97}^{+5.78} & 23.92_{-2.44}^{+0.46} \\ \text { 12II } & 0.91 ; 0.10 & 98.17_{-0.06}^{+0.07} & 1.93_{-0.10}^{+0.39} & 26.25_{-2.50}^{+4.50} & 18.17_{-1.05}^{+1.29} \\ \text { 12III } & 0.95 ; 0.05 & 48.88_{-0.04}^{+0.03} & 2.70_{-0.37}^{+0.47} & 25.32_{-3.83}^{+9.70} & 12.70_{-0.30}^{+0.31} \\ \text { 9I } & 0.87 ; 0.15 & 147.01_{-0.03}^{+0.13} & 1.73_{-0.07}^{+0.10} & 29.97_{-1.97}^{+2.02} & 21.22_{-0.37}^{+0.54} \\ \text { 9II } & 0.91 ; 0.10 & 97.95_{-0.03}^{+0.09} & 2.00_{-0.17}^{+0.17} & 25.65_{-2.15}^{+2.35} & 16.56_{-0.64}^{+0.57} \\ \text { 9III } & 0.95 ; 0.05 & 48.86_{-0.03}^{+0.14} & 2.70_{-0.37}^{+1.30} & 24.01_{-3.89}^{+9.49} & 11.67_{-0.84}^{+0.40} \\ \text { 6I } & 0.87 ; 0.15 & 147.15_{-0.07}^{+0.02} & 1.67_{-0.17}^{+0.17} & 27.08_{-1.82}^{+0.93} & 18.95_{-1.07}^{+0.62} \\ \text { 6II } & 0.91 ; 0.10 & 98.24_{-0.01}^{+0.05} & 1.88_{-0.22}^{+0.12} & 23.75_{-3.25}^{+3.25} & 14.93_{-0.91}^{+0.85} \\ \text { 6III } & 0.95 ; 0.05 & 49.05_{-0.04}^{+0.03} & 2.43_{-0.10}^{+0.40} & 22.15_{-2.15}^{+4.35} & 11.01_{-0.96}^{+1.08} \\ \text { 3I } & 0.87 ; 0.15 & 147.09_{-0.06}^{+0.05} & 1.70_{-0.03}^{+0.13} & 24.70_{-0.70}^{+1.05} & 18.15_{-0.40}^{+0.64} \\ \text { 3II } & 0.91 ; 0.10 & 98.07_{-0.03}^{+0.08} & 1.80_{-0.12}^{+0.03} & 21.60_{-1.08}^{+0.39} & 14.67_{-0.29}^{+0.13} \\ \text { 3III } & 0.95 ; 0.05 & 48.98_{-0.04}^{+0.03} & 2.43_{-0.10}^{+0.07} & 20.50_{-1.75}^{+1.25} & 10.70_{-0.26}^{+0.51} \\ \text { 0I } & 0.87 ; 0.15 & 147.14_{-0.02}^{+0.05} & 1.80_{-0.13}^{+0.20} & 21.70_{-1.45}^{+1.53} & 17.13_{-0.17}^{+0.45} \\ \text { 0II } & 0.91 ; 0.10 & 98.24_{-0.03}^{+0.05} & 2.17_{-0.17}^{+0.17} & 17.65_{-1.40}^{+2.10} & 13.40_{-0.35}^{+0.48} \\ \text { 0III } & 0.95 ; 0.05 & 48.87_{-0.04}^{+0.03} & 2.70_{-0.03}^{+0.13} & 14.95_{-1.20}^{+1.05} & 10.01_{-0.39}^{+0.75}\end{array}$

Table 1. Table showing operational parameters, including the slope angle $\theta$, density ratio $\gamma$, relative density difference $\epsilon=(1-\gamma) / \gamma$, reduced gravity $g_{0}^{\prime}$, time $t_{\max }$ and front location $x_{f_{\max }}$, measured from the lock gate, at which the gravity currents reach the maximum front velocity $U_{f_{\max }}$. Each value is the average of five experiments. The error estimates are to add and subtract the maximum and minimum values and are not the root-mean-square estimates.

gravity current moves into the deceleration phase. The gravity current takes a wedge shape, which grows in width and height as the gravity current propagates downslope, as shown in figures 2 and 3 at $t=2,8 \mathrm{~s}$. As shown in figure 2 at $t=15,20 \mathrm{~s}$, an 'active' part of the wedge separates from the body of the current and leaves the inactive part moving slowly behind. The side view images show the edge of the 'active' part of the wedge is uplifted, in that the interface between the 'active' part of the wedge and ambient fluid is raised, in the final stage of propagation. Our observation on the separation of the 'active' head and mixing with the ambient fluid in the final stage of propagation is persistent for the gravity currents on an unbounded uniform $12^{\circ}$ slope at all relative density differences, including $\epsilon \approx 0.15,0.10,0.05(\gamma \approx 0.87,0.91,0.95)$. Such an observation for the non-Boussinesq gravity currents is similar to the Boussinesq case (Dai \& Huang 2020), but holds a clue to the possibility that the non-Boussinesq gravity currents on an unbounded uniform $12^{\circ}$ slope may have become Boussinesq ones in the final stage of propagation, as we will discuss later. Other top view and side view images for the gravity currents at $\epsilon \approx 0.10$, $0.05(\gamma \approx 0.91,0.95)$ are qualitatively similar and are omitted here for brevity.

\subsubsection{Quantitative results}

From the side view images of the gravity current, as shown in figure 3, the front location can be extracted as the furthest location reached by the gravity current and the front velocity can be calculated. Figure 4 shows the front location and front velocity histories 
(a) 80

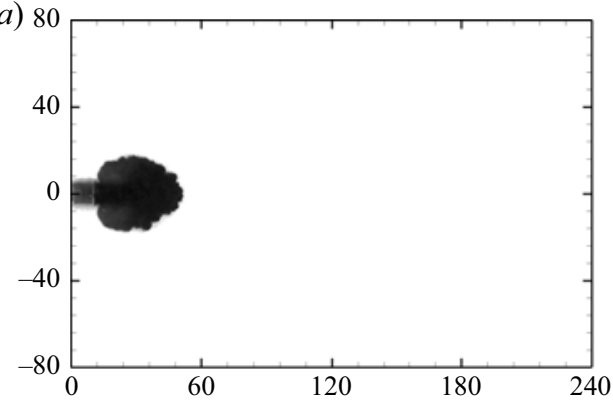

(b) 80

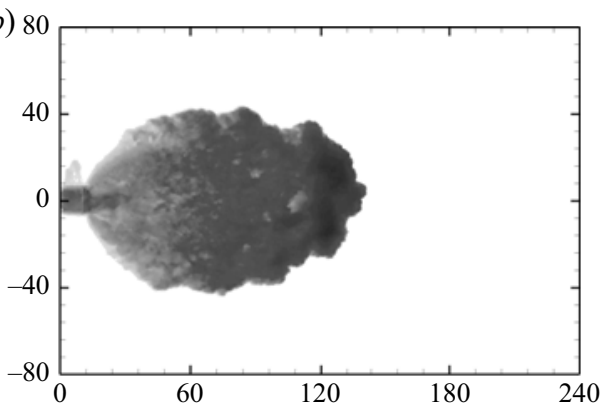

(c)

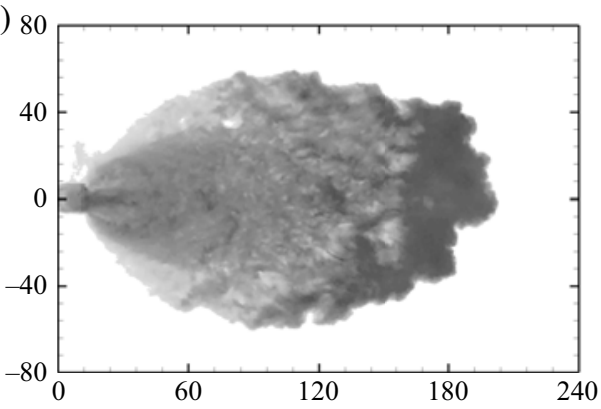

$(d)$

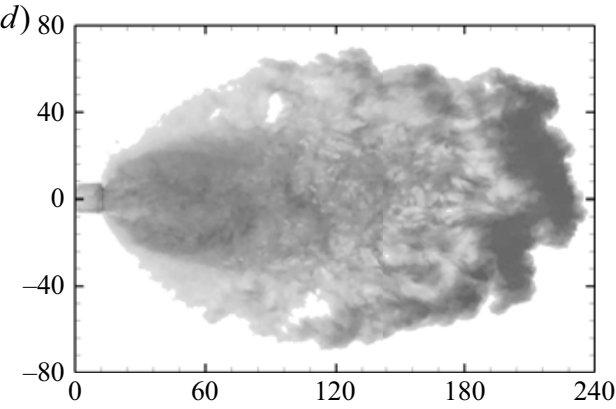

Figure 2. Experiment 04/09/17-2: top view images for the gravity current on an unbounded uniform $12^{\circ}$ slope. The reduced gravity of the heavy fluid in the lock was $g_{0}^{\prime}=147.15 \mathrm{~cm} \mathrm{~s}^{-2}$, i.e. $\epsilon \approx 0.15(\gamma \approx 0.87)$. Distances in the downslope and spanwise directions are in units of $\mathrm{cm}$. Time instances are chosen at $(a-d) t=2,8,15$, $20 \mathrm{~s}$.

(a) 20

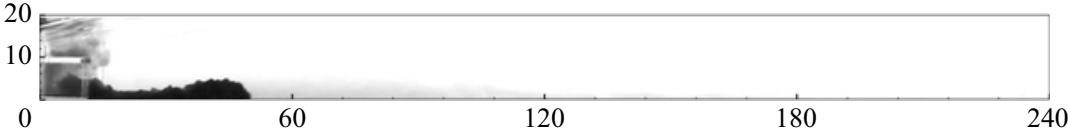

(b)

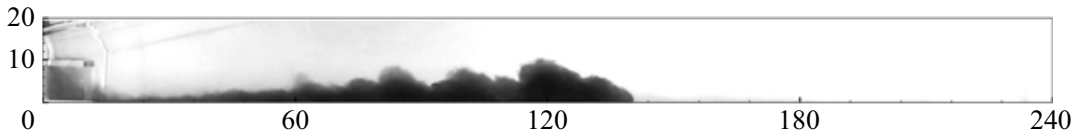

(c)

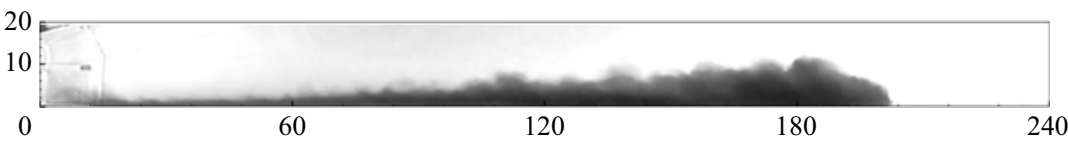

(d)

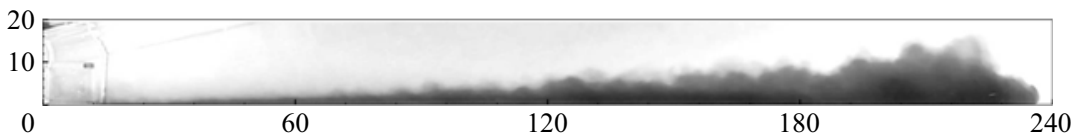

Figure 3. Experiment 04/09/17-2: side view images for the gravity current on an unbounded uniform $12^{\circ}$ slope as shown in figure 2. Distances in the downslope and wall-normal directions are in units of $\mathrm{cm}$. Time instances are chosen at $(a-d) t=2,8,15,20 \mathrm{~s}$.

for the gravity current at $\epsilon \approx 0.15(\gamma \approx 0.87)$ propagating on an unbounded uniform $12^{\circ}$ slope. From the front velocity history, it is observed that the gravity current moves into the deceleration phase after reaching its maximum front velocity $U_{f_{\max }} \approx 24.38 \mathrm{~cm} \mathrm{~s}^{-1}$ at $t \approx 1.67 \mathrm{~s}$. 
(a)

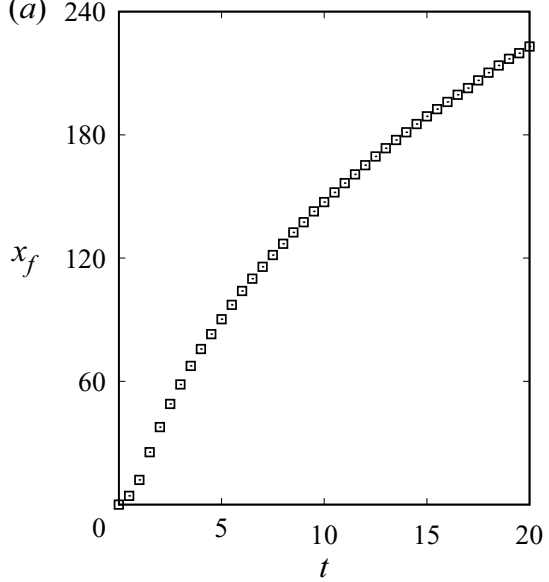

(b)

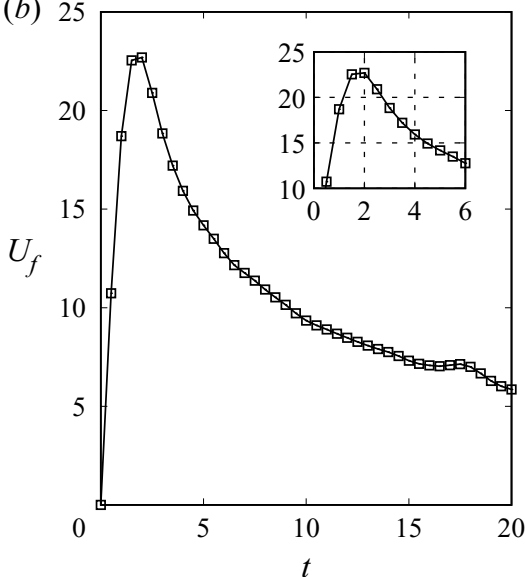

Figure 4. Experiment 04/09/17-2: front location history $(a)$ and front velocity history $(b)$ for the gravity current propagating on an unbounded uniform $12^{\circ}$ slope. The reduced gravity of the heavy fluid in the lock was $g_{0}^{\prime}=147.15 \mathrm{~cm} \mathrm{~s}^{-2}$. The maximum front velocity $U_{f_{\max }} \approx 24.38 \mathrm{~cm} \mathrm{~s}^{-1}$ occurs at $t \approx 1.67 \mathrm{~s}$. The front location is in units of $\mathrm{cm}$, front velocity is in units of $\mathrm{cm} \mathrm{s}^{-1}$ and time is in units of $\mathrm{s}$.

To examine the front location history in the early and late stages of the deceleration phase, we plot the front location and time in terms of $\left(x_{f}+x_{0}\right)^{2}$ vs $t$ in figure $5(a)$ and $\left(x_{f}+x_{0}\right)^{8 / 3}$ vs $t$ in figure $5(b)$. Here, $\left(x_{f}+x_{0}\right)$ represents the front location measured from the virtual origin, which can be identified by extrapolating the width of the wedge in the upslope direction, as shown in figure 1. In the early deceleration phase, the gravity current propagates downslope with an integrated wedge shape and figure 5(a) reveals that the front location history follows the power relationship (2.9) during $5 \lesssim t \lesssim 10 \mathrm{~s}$. In the late deceleration phase, an 'active' head separates from the body of the current and figure $5(b)$ reveals that the front location history follows the power relationship (2.11) during $t \gtrsim 10 \mathrm{~s}$.

Based on the slope of the best fit to the early deceleration phase and the buoyancy $B=117720.00 \mathrm{~cm}^{4} \mathrm{~s}^{-2}$, the experimental constant $K_{I}=111.80$ is calculated according to (2.9). The entrainment coefficient $\alpha=0.149$ is then calculated based on the experimental constant $K_{I}$ and (2.10). The front location history begins to deviate from the power relationship (2.9) at $t \gtrsim 10 \mathrm{~s}$, when the front Reynolds number based on the front velocity and front thickness, $R e_{f}=U_{f} h / v$, is approximately $R e_{f} \approx 6000$. Based on the slope of the best fit to the late deceleration phase, the experimental constant $K_{V S}=3.49$ is calculated according to (2.11). Other dependent variables, including the experimental constant $K_{I}$, the entrainment coefficient $\alpha$ and the experimental constant $K_{V S}$, for the gravity currents propagating on unbounded uniform $12^{\circ}, 9^{\circ}$ and $6^{\circ}$ slopes are all listed in table 2 .

\subsection{Non-Boussinesq gravity currents on unbounded uniform $9^{\circ}$ and $6^{\circ}$ slopes}

Non-Boussinesq gravity currents propagating on unbounded uniform $9^{\circ}$ and $6^{\circ}$ slopes are qualitatively similar to the non-Boussinesq gravity currents propagating on an unbounded uniform $12^{\circ}$ slope and their images are omitted for brevity. We summarise the influence of the slope angle and the relative density difference for the non-Boussinesq gravity currents on unbounded uniform $9^{\circ}$ and $6^{\circ}$ slopes and the readers are referred to tables 1 and 2 for other quantitative measures. 

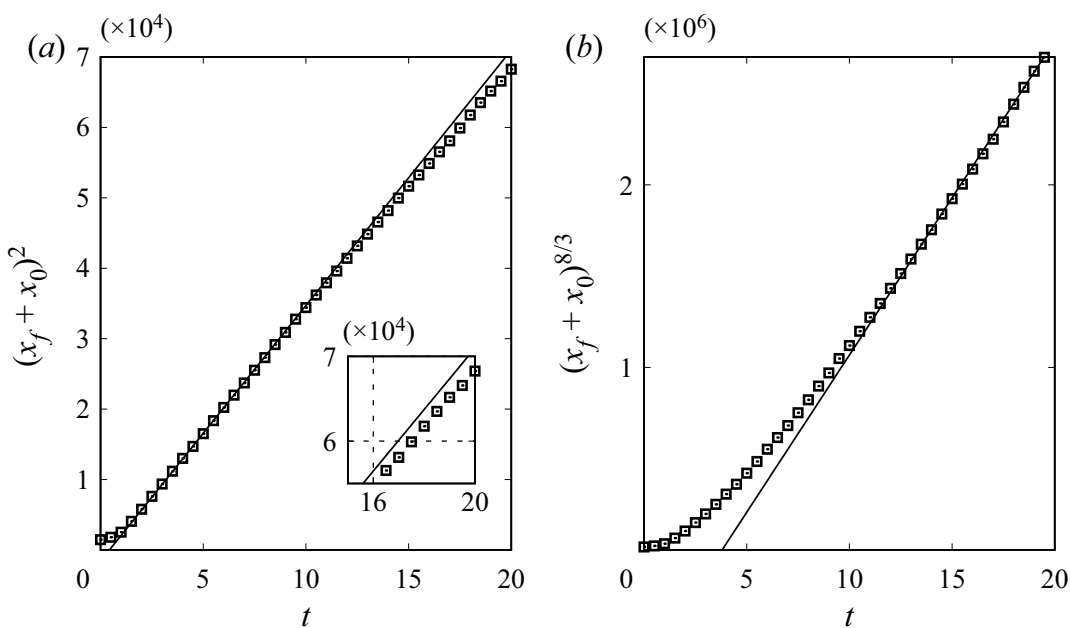

Figure 5. Experiment 04/09/17-2: relationship between $(a)\left(x_{f}+x_{0}\right)^{2}$ and $t$ and $(b)\left(x_{f}+x_{0}\right)^{8 / 3}$ and $t$ for the gravity current propagating on an unbounded uniform $12^{\circ}$ slope. The reduced gravity of the heavy fluid in the lock was $g_{0}^{\prime}=147.15 \mathrm{~cm} \mathrm{~s}^{-2}$. The front location is in units of $\mathrm{cm}$ and time is in units of $\mathrm{s}$. The solid line in $(a)$ represents the straight line of best fit to the early deceleration phase and the fitting equation is $\left(x_{f}+x_{0}\right)^{2}=\left(K_{I} B\right)^{1 / 2}\left(t+t_{I}\right)$, where $K_{I}=111.80, B=117720.00 \mathrm{~cm}^{4} \mathrm{~s}^{-2}, x_{0}=38.27 \mathrm{~cm}$ and $t_{I}=-0.43$ $\mathrm{s}$. The solid line in $(b)$ represents the straight line of best fit to the late decelearation phase and the fitting equation is $\left(x_{f}+x_{0}\right)^{8 / 3}=K_{V S} B^{2 / 3} V_{0}^{2 / 9} v^{-1 / 3}\left(t+t_{V S}\right)$, where $K_{V S}=3.49$ and $t_{V S}=-4.72 \mathrm{~s}$. The maximum front velocity $U_{f_{\max }} \approx 24.38 \mathrm{~cm} \mathrm{~s}^{-1}$ occurs at $t \approx 1.67 \mathrm{~s}$.

$\begin{array}{lcccccc}\text { Case } & \alpha & x_{0}(\mathrm{~cm}) & K_{I} & t_{I}(\mathrm{~s}) & K_{V S} & t_{V S}(\mathrm{~s}) \\ \text { 12I } & 0.145_{-0.018}^{+0.007} & 39.18_{-9.18}^{+6.30} & 122.53_{-15.86}^{+44.84} & -0.57_{-0.68}^{+0.84} & 4.04_{-0.550}^{+0.613} & -4.89_{-1.48}^{+1.61} \\ \text { 12II } & 0.152_{-0.008}^{+0.009} & 41.54_{-6.54}^{+5.45} & 110.68_{-8.27}^{+10.74} & -0.56_{-0.52}^{+0.53} & 3.91_{-0.425}^{+0.464} & -6.35_{-1.79}^{+1.04} \\ \text { 12III } & 0.157_{-0.007}^{+0.015} & 42.05_{-3.05}^{+1.17} & 104.00_{-24.04}^{+20.81} & -0.75_{-0.97}^{+1.3} & 4.14_{-0.425}^{+0.439} & -7.34_{-2.08}^{+1.52} \\ \text { 9I } & 0.158_{-0.009}^{+0.011} & 40.98_{-3.28}^{+6.31} & 99.36_{-15.60}^{+14.61} & -0.23_{-0.33}^{+0.17} & 3.67_{-0.247}^{+0.273} & -5.53_{-1.36}^{+1.22} \\ \text { 9II } & 0.164_{-0.010}^{+0.012} & 44.44_{-2.84}^{+3.27} & 91.28_{-14.17}^{+13.75} & -0.32_{-1.75}^{+0.73} & 3.78_{-0.375}^{+0.458} & -7.06_{-1.53}^{+1.54} \\ \text { 9III } & 0.171_{-0.006}^{+0.005} & 45.46_{-7.87}^{+7.20} & 84.39_{-5.18}^{+6.05} & -0.57_{-4.44}^{+1.02} & 3.61_{-0.562}^{+0.557} & -7.05_{-2.64}^{+3.06} \\ \text { 6I } & 0.183_{-0.017}^{+0.019} & 45.92_{-4.12}^{+5.77} & 72.51_{-14.87}^{+17.36} & 0.41_{-0.41}^{+0.35} & 2.89_{-0.643}^{+0.361} & -4.39_{-2.23}^{+4.06} \\ \text { 6II } & 0.193_{-0.011}^{+0.026} & 47.38_{-4.38}^{+4.31} & 64.36_{-16.01}^{+8.54} & 0.72_{-0.93}^{+0.54} & 2.81_{-0.250}^{+0.266} & -5.41_{-2.12}^{+3.49} \\ \text { 6III } & 0.201_{-0.021}^{+0.020} & 49.11_{-8.08}^{+4.88} & 61.03_{-8.26}^{+13.58} & 0.95_{-0.82}^{+1.14} & 2.70_{-0.279}^{+0.366} & -5.14_{-4.20}^{+5.60}\end{array}$

Table 2. Table showing the dependent variables for gravity currents propagating on unbounded uniform $12^{\circ}$, $9^{\circ}, 6^{\circ}$ slopes, including the entrainment coefficient $\alpha$, distance from the virtual origin to the lock gate $x_{0}$, experimental constant $K_{I}, t$ intercept $t_{I}$ in (2.9), experimental constant $K_{V S}$ and $t$ intercept $t_{V S}$ in (2.11). The subscripts $I$ and $V S$ represent the inertial phase and viscous phase on the steeper slopes in our study $\left(12^{\circ}, 9^{\circ}\right.$, $\left.6^{\circ}\right)$, respectively. Each value is the average of five experiments. The error estimates are to add and subtract the maximum and minimum values and are not the root-mean-square estimates.

Figure 6 shows the experimental constant $K_{I}$ against the slope angle at different values of relative density difference. In our slope angle range, based on our experiments and Ross et al. (2002), it can be concluded that the experimental constant $K_{I}$ increases as the 


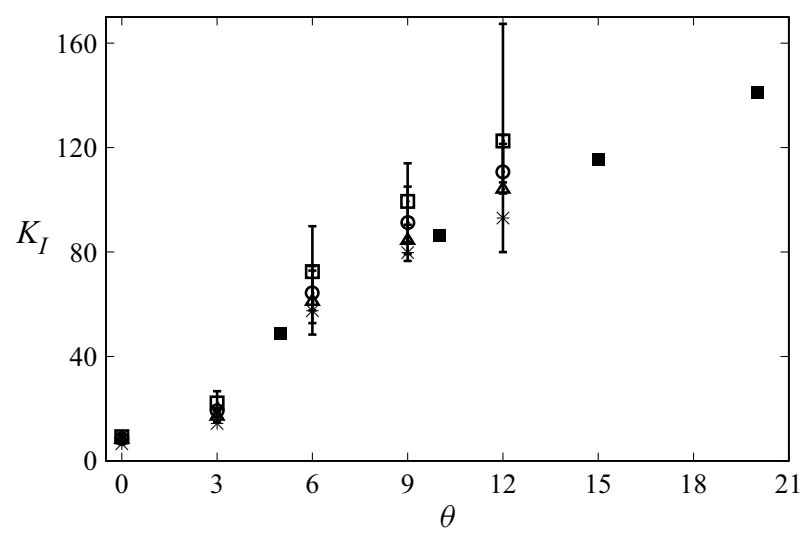

Figure 6. Dimensionless constant $K_{I}$ as a function of the slope angle $\theta$ and the relative density difference $\epsilon$. Symbols: $\square, \epsilon \approx 0.15(\gamma \approx 0.87) ; \circ, \epsilon \approx 0.10(\gamma \approx 0.91) ; \Delta, \epsilon \approx 0.05(\gamma \approx 0.95) ; *, \epsilon \approx 0.02(\gamma \approx 0.98)$ reported by Dai \& Huang (2020); $\boldsymbol{\square}$, values reported by Ross et al. (2002), in which $0.011 \leq \epsilon \leq 0.039$ $(0.962 \leq \gamma \leq 0.989)$.

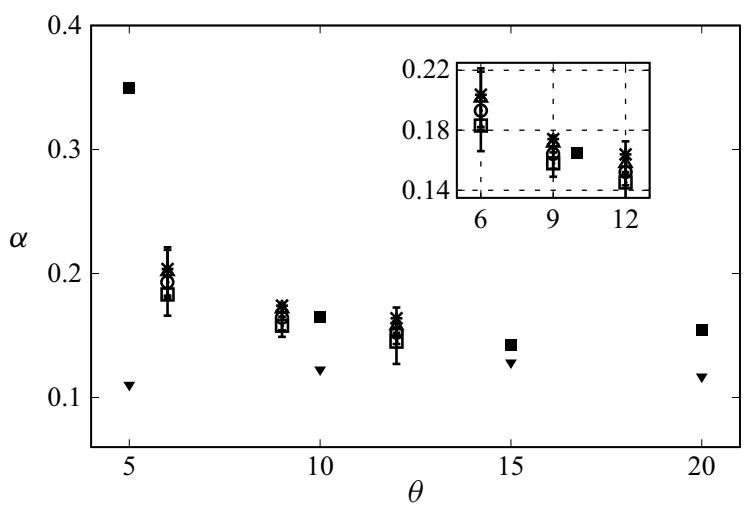

Figure 7. Entrainment coefficient $\alpha$ as a function of the slope angle $\theta$ and the relative density difference $\epsilon$. Symbols: $\square, \epsilon \approx 0.15(\gamma \approx 0.87) ; \circ, \epsilon \approx 0.10(\gamma \approx 0.91) ; \triangle, \epsilon \approx 0.05(\gamma \approx 0.95) ; *, \epsilon \approx 0.02(\gamma \approx 0.98)$ reported by Dai \& Huang (2020); $\mathbf{\square}$, values reported by Ross et al. (2002), in which $0.011 \leq \epsilon \leq 0.039$ $(0.962 \leq \gamma \leq 0.989) ; \boldsymbol{\nabla}$, values reported by Zgheib et al. (2016).

slope angle increases and as the relative density difference increases. Figure 7 shows the entrainment coefficient $\alpha$ against the slope angle at different values of relative density difference. It is found that the entrainment coefficient also depends on the slope angle and decreases as the relative density difference increases, as shown in figure 7 and the inset. It is worth noting that the gravity currents propagating on unbounded uniform $12^{\circ}, 9^{\circ}, 6^{\circ}$ slopes maintain a wedge shape in the early deceleration phase and we may use the wedge integral model (2.10) to calculate the entrainment coefficient. As will be shown later, the gravity currents propagating on an unbounded uniform $3^{\circ}$ slope and on an unbounded horizontal boundary do not take the wedge shape and we shall not use the wedge integral model to calculate the entrainment coefficient for the gravity currents on an unbounded uniform $3^{\circ}$ slope and on an unbounded horizontal boundary.

As listed in table 2 and shown in figure 8, the experimental constant $K_{V S}$ increases as the slope angle increases but, surprisingly, appears not to be strongly influenced by the relative density difference. As shown in figure 8 , for $0.05 \leq \epsilon \leq 0.15, K_{V S}$ varies erratically in 


\section{Gravity currents on unbounded uniform slopes}

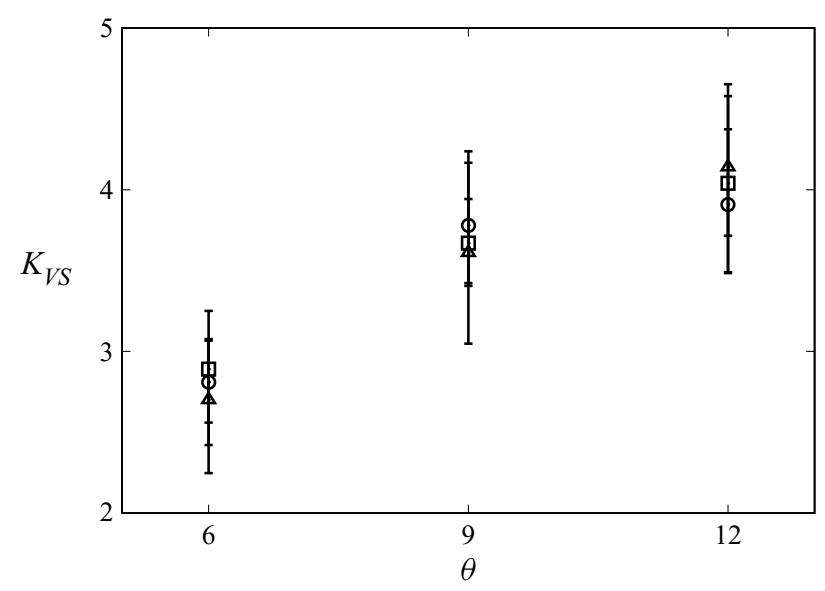

Figure 8. Dimensionless constant $K_{V S}$ as a function of the slope angle $\theta$. The influence of the relative density difference on $K_{V S}$ is not significant in the range $0.05 \leq \epsilon \leq 0.15$. Symbols: $\square, \epsilon \approx 0.15(\gamma \approx 0.87) ; \circ, \epsilon \approx$ $0.10(\gamma \approx 0.91) ; \Delta, \epsilon \approx 0.05(\gamma \approx 0.95)$.

(a)

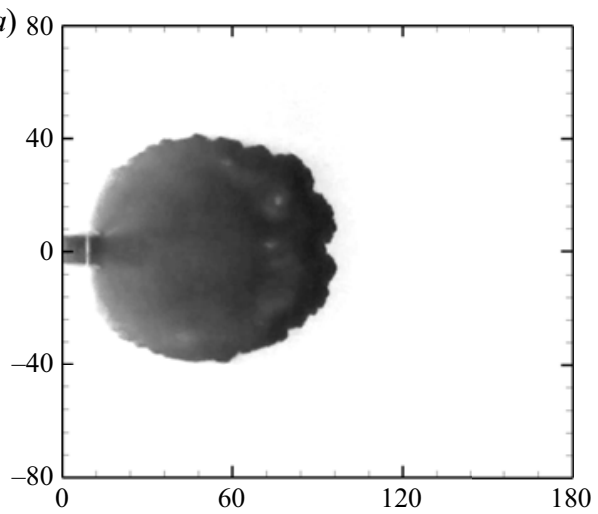

(c)

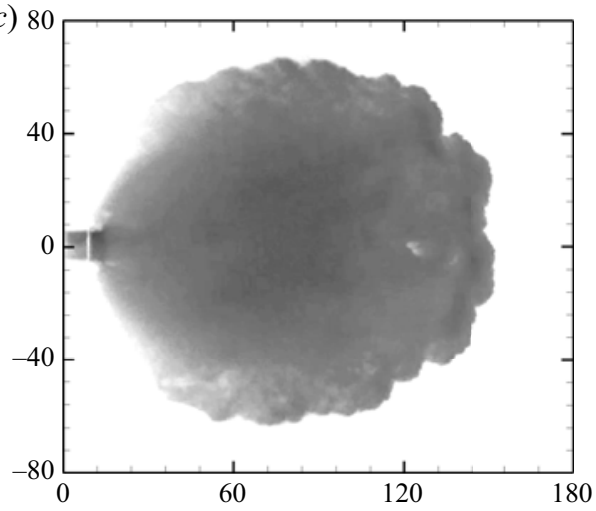

(b)

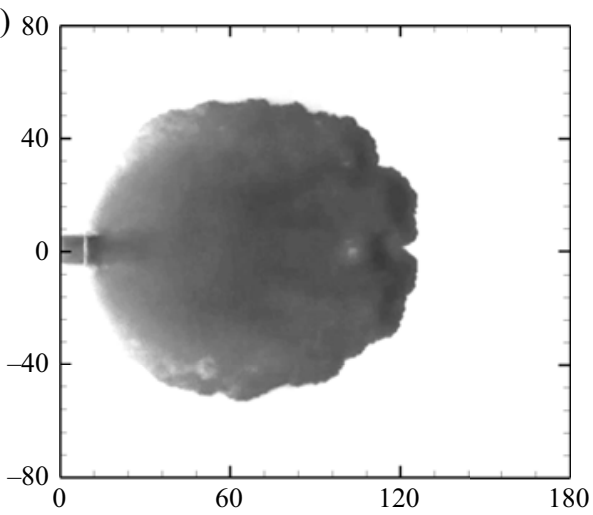

(d) 80

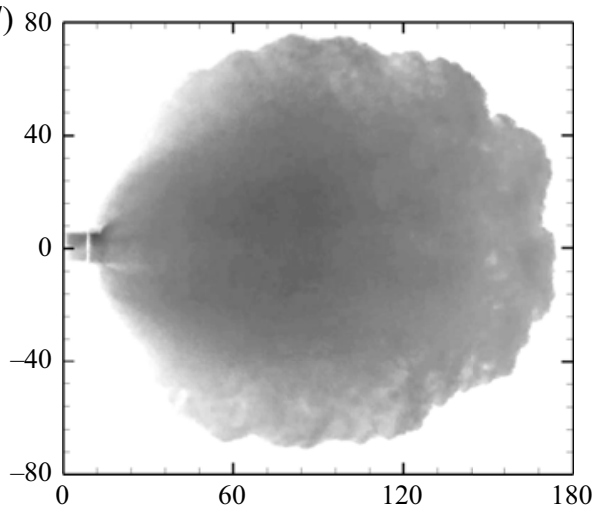

Figure 9. Experiment 07/26/17-2: top view images of the gravity current propagating on an unbounded uniform $3^{\circ}$ slope. The reduced gravity of the heavy fluid in the lock was $g_{0}^{\prime}=147.15 \mathrm{~cm} \mathrm{~s}^{-2}$, i.e. $\epsilon \approx 0.15$ $(\gamma \approx 0.87)$. Distances in the downslope and spanwise directions are in units of $\mathrm{cm}$. Time instances are chosen at $(a-d) t=6,10,15,20 \mathrm{~s}$. In this experiment, the maximum front velocity $U_{f_{\max }} \approx 18.00 \mathrm{~cm} \mathrm{~s}^{-1}$ occurs at $t \approx 1.67 \mathrm{~s}$. 


\section{A. Dai and Y.-L. Huang}

(a) 20

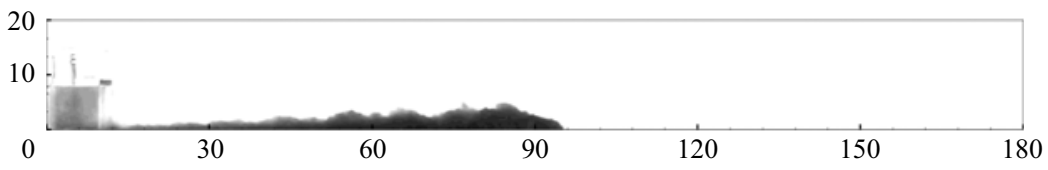

(b) 20

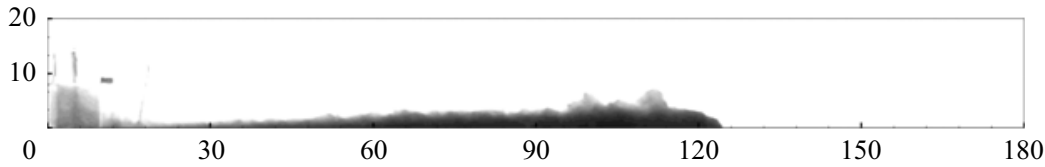

(c) 20

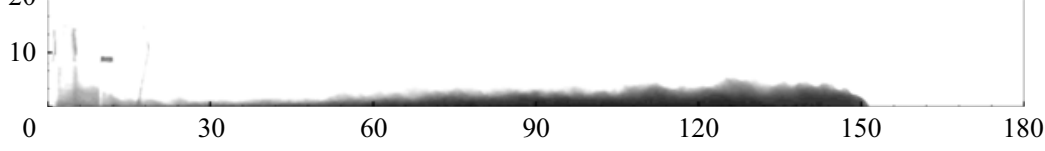

(d) 20

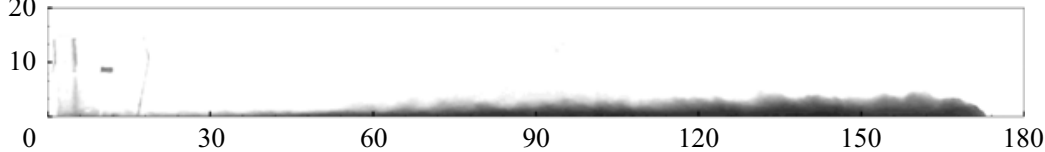

Figure 10. Experiment 07/26/17-2: side view images for the gravity current propagating on an unbounded uniform $3^{\circ}$ slope as shown in figure 9 . Distances in the downslope and wall-normal directions are in units of $\mathrm{cm}$. Time instances are chosen at $(a-d) t=6,10,15,20 \mathrm{~s}$.

the range $3.49 \leq K_{V S} \leq 4.66$ at $\theta=12^{\circ}, 3.05 \leq K_{V S} \leq 4.24$ at $\theta=9^{\circ}$ and $2.25 \leq K_{V S} \leq$ 3.26 at $\theta=6^{\circ}$. The fact that the influence of the relative density difference on $K_{V S}$ is not significant suggests that the non-Boussinesq gravity currents on unbounded uniform $12^{\circ}$, $9^{\circ}$ and $6^{\circ}$ slopes may have become Boussinesq ones in the late deceleration phase.

\subsection{Non-Boussinesq gravity currents on an unbounded uniform $3^{\circ}$ slope}

Non-Boussinesq gravity currents propagating on an unbounded uniform $3^{\circ}$ slope are qualitatively different from the non-Boussinesq gravity currents on unbounded uniform $12^{\circ}, 9^{\circ}$ and $6^{\circ}$ slopes. The gravity current maintains a shape more akin to a disk even in the late deceleration phase, as shown by the top view and side view images in figures 9 and 10 .

In the early deceleration phase, figure 11(a) shows that the front location history follows the power relationship (2.9) during $5 \lesssim t \lesssim 15$ s and we may calculate the experimental constant $K_{I}=26.70$, which is listed in table 3 . At $t \gtrsim 15 \mathrm{~s}$, the front location history begins to deviate from the power relationship (2.9) when the front Reynolds number is approximately $R e_{f} \approx 2000$. In the late deceleration phase, figure 11(b) shows that the front location history follows the power relationship (2.12) during $t \gtrsim 15 \mathrm{~s}$ and we may calculate the experimental constant $K_{V M}=94.37$, which is also listed in table 3 . The experimental constant $K_{V M}$ is evidently not only a function of the slope angle but also a function of the relative density difference. The influence of the relative density difference is carried along into $K_{I}$ during the early deceleration phase and into $K_{V M}$ during the late deceleration phase for the gravity currents on an unbounded uniform $3^{\circ}$ slope.

\subsection{Non-Boussinesq gravity currents on an unbounded horizontal boundary}

We also performed the experiments on the gravity currents on an unbounded horizontal boundary, of which the morphology (not shown) is qualitatively similar to the gravity current on an unbounded uniform $3^{\circ}$ slope. 

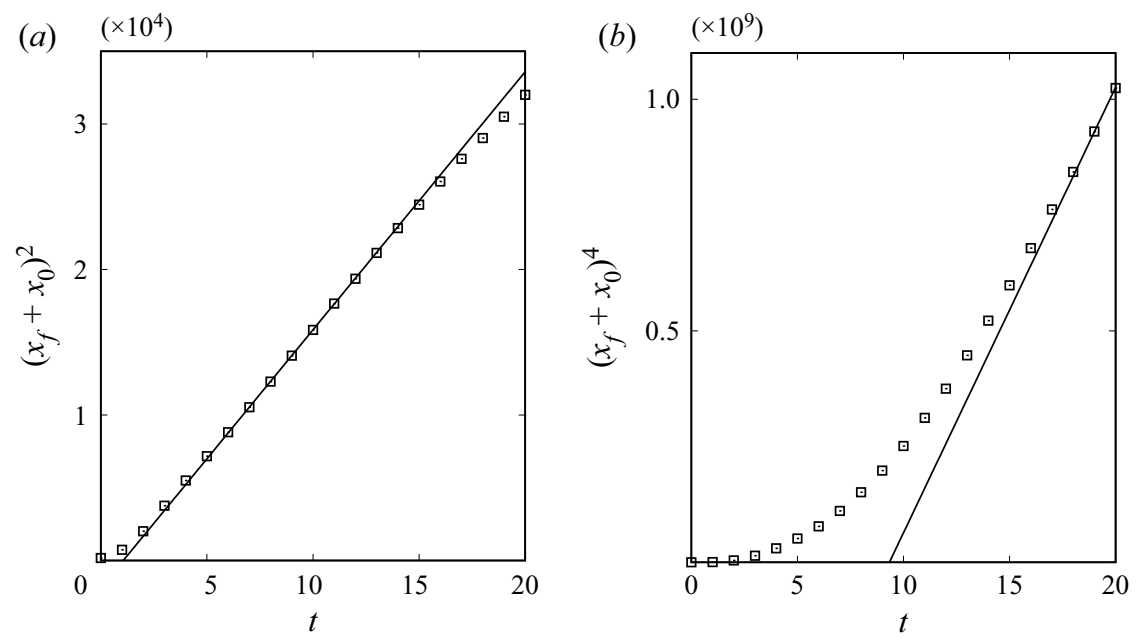

Figure 11. Experiment 07/26/17-2: relationship between $(a)\left(x_{f}+x_{0}\right)^{2}$ and $t$ and $(b)\left(x_{f}+x_{0}\right)^{4}$ and $t$ for the gravity current propagating on an unbounded uniform $3^{\circ}$ slope. The reduced gravity of the heavy fluid in the lock was $g_{0}^{\prime}=146.87 \mathrm{~cm} \mathrm{~s}^{-2}$. The front location is in units of $\mathrm{cm}$ and time is in units of s. The solid line in $(a)$ represents the straight line of best fit to the early deceleration phase and the fitting equation is $\left(x_{f}+x_{0}\right)^{2}=\left(K_{I} B\right)^{1 / 2}\left(t+t_{I}\right)$, where $K_{I}=26.70, B=117720.00 \mathrm{~cm}^{4} \mathrm{~s}^{-2}, x_{0}=13.15 \mathrm{~cm}$ and $t_{I}=-1.05 \mathrm{~s}$. The solid line in $(b)$ represents the straight line of best fit to the late deceleration phase and the fitting equation is $\left(x_{f}+x_{0}\right)^{4}=K_{V M} B^{2 / 3} V_{0}^{2 / 3} v^{-1 / 3}\left(t+t_{V M}\right)$, where $K_{V M}=94.37$ and $t_{V M}=-8.67 \mathrm{~s}$. The maximum front velocity $U_{f_{\text {max }}} \approx 18.00 \mathrm{~cm} \mathrm{~s}^{-1}$ occurs at $t \approx 1.67 \mathrm{~s}$.

$\begin{array}{lccccc}\text { Case } & x_{0}(\mathrm{~cm}) & K_{I} & t_{I}(\mathrm{~s}) & K_{V M} & t_{V M}(\mathrm{~s}) \\ \text { 3I } & 9.08_{-2.26}^{+4.06} & 22.20_{-5.07}^{+4.49} & -1.05_{-0.26}^{+0.27} & 85.29_{-8.12}^{+9.07} & -9.24_{-2.14}^{+0.89} \\ \text { 3II } & 11.91_{-4.34}^{+4.37} & 19.22_{-2.46}^{+1.09} & -1.42_{-0.28}^{+0.22} & 75.05_{-7.56}^{+5.05} & -12.02_{-0.23}^{+0.17} \\ \text { 3III } & 9.06_{-4.63}^{+5.51} & 17.02_{-2.23}^{+1.99} & -1.05_{-1.02}^{+1.17} & 69.47_{-4.60}^{+4.95} & -15.55_{-1.96}^{+2.45} \\ \text { 0I } & 7.98_{-5.10}^{+4.17} & 9.24_{-1.16}^{+0.76} & 0.34_{-0.99}^{+1.60} & 18.29_{-4.58}^{+3.28} & -4.32_{-1.67}^{+1.52} \\ \text { OII } & 7.19_{-1.34}^{+3.25} & 8.52_{-0.49}^{+0.78} & -1.20_{-0.37}^{+0.49} & 14.11_{-2.54}^{+2.98} & -7.31_{-2.31}^{+1.86} \\ \text { OIII } & 5.73_{-3.48}^{+4.91} & 8.29_{-1.45}^{+2.23} & -1.96_{-0.61}^{+0.49} & 11.47_{-2.23}^{+4.39} & -5.91_{-5.57}^{+3.86}\end{array}$

Table 3. Table showing the dependent variables for gravity currents propagating on a $3^{\circ}$ unbounded uniform slope and on an unbounded horizontal boundary, including the distance from the virtual origin to the lock gate $x_{0}$, experimental constants $K_{I}, t$ intercept $t_{I}$ in (2.9), experimental constant $K_{V M}$ and $t$ intercept $t_{V M}$ in (2.12). The subscripts $I$ and $V M$ represent the inertial phase and viscous phase on the milder slopes in our study $\left(3^{\circ}\right.$ and $0^{\circ}$ ), respectively. Each value is the average of five experiments. The error estimates are to add and subtract the maximum and minimum values and are not the root-mean-square estimates.

For the gravity currents on an unbounded horizontal boundary, the experimental constant $K_{I}$ tends to increase as the relative density difference increases, as shown in table 3. In the late deceleration phase, the experimental constant $K_{V M}$ is a function of both the slope angle and the relative density difference, as listed in table 3 and shown in figure 12. The influence of the relative density difference is carried along into the late deceleration phase on $K_{V M}$ for the gravity currents on an unbounded horizontal boundary. Our observations suggest that the non-Boussinesq gravity currents on an 


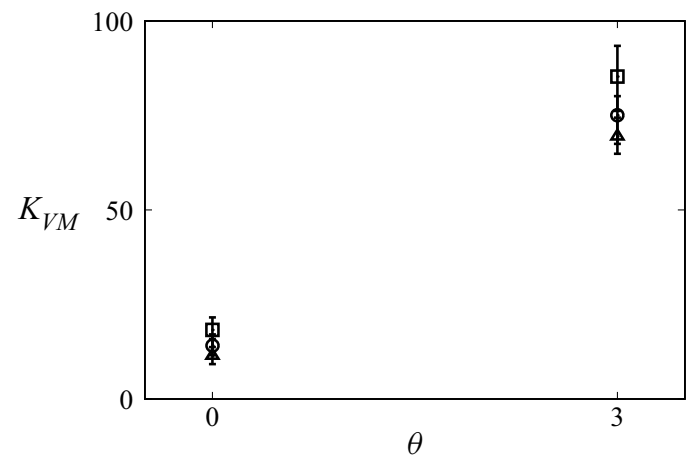

Figure 12. Dimensionless constant $K_{V M}$ as a function of the slope angle $\theta$ and the relative density difference $\epsilon$. The influence of the relative density difference on $K_{V M}$ is clear, as also shown in table 3 . Symbols: $\square, \epsilon \approx 0.15$ $(\gamma \approx 0.87) ; \circ, \epsilon \approx 0.10(\gamma \approx 0.91) ; \Delta, \epsilon \approx 0.05(\gamma \approx 0.95)$.

unbounded uniform $3^{\circ}$ slope and on an unbounded horizontal boundary may still remain non-Boussinesq ones in the late deceleration phase.

\section{Conclusions}

Experiments on the Boussinesq and non-Boussinesq gravity currents produced from a finite volume of heavy fluid propagating into an environment of light ambient fluid on unbounded uniform slopes in the range $0^{\circ} \leq \theta \leq 12^{\circ}$ are presented. The relative density difference covers the Boussinesq and non-Boussinesq cases in the range $0.05 \leq \epsilon \leq 0.15$ in this study. After the lock gate is removed, the gravity currents go through an acceleration phase and move into the early and late stages of the deceleration phase. Our focus in this study is on the influence of the relative density difference on the deceleration phase of the propagation.

For the gravity currents propagating on unbounded uniform $12^{\circ}, 9^{\circ}$ and $6^{\circ}$ slopes, in the early deceleration phase, the gravity currents take a wedge shape and the front location history follows the power relationship (2.9), in which the dimensionless constant $K_{I}$ is a function of both the slope angle and the relative density difference. In the late deceleration phase, an 'active' head separates from the body of the current and mixes with the ambient fluid. The front location history in the late deceleration phase follows the power relationship (2.11), in which the dimensionless constant $K_{V S}$ is influenced by the slope angle but not significantly influenced by the relative density difference. The observation that the relative density difference has no significant influence on the dimensionless constant $K_{V S}$ in the late deceleration phase suggests that the non-Boussinesq gravity currents on unbounded uniform $12^{\circ}, 9^{\circ}$ and $6^{\circ}$ slopes may have become Boussinesq ones in the late deceleration phase.

For the gravity currents propagating on an unbounded uniform $3^{\circ}$ slope and on an unbounded horizontal boundary, the gravity currents maintain an integrated disk shape throughout the motion. In the early deceleration phase, the front location history follows the power relationship (2.9) and the dimensionless constant $K_{I}$ is a function of both the slope angle and the relative density difference. In the late deceleration phase, the gravity currents still maintain an integrated disk shape without the separation of an 'active' head from the body of the gravity currents. The front location history in the late deceleration phase follows the power relationship (2.12), in which the dimensionless constant $K_{V M}$ is influenced by both the slope angle and the relative density difference. The influence of the relative density difference is carried along into the late deceleration phase on $K_{V M}$ and 
such an observation suggests that the non-Boussinesq gravity currents on an unbounded uniform $3^{\circ}$ slope and on an unbounded uniform horizontal boundary may still remain non-Boussinesq ones in the late deceleration phase.

Our experiments indicate that, depending on the slope angle, two different flow morphologies are possible concerning the final stage of the non-Boussinesq gravity currents propagating on unbounded uniform slopes. More importantly, our results further indicate that the non-Boussinesq gravity currents on the milder unbounded uniform slopes in this study $\left(3^{\circ}\right.$ and $\left.0^{\circ}\right)$ may remain non-Boussinesq ones in the late deceleration phase while the non-Boussinesq gravity currents on the steeper unbounded uniform slopes in this study $\left(12^{\circ}, 9^{\circ}\right.$ and $\left.6^{\circ}\right)$ may have become Boussinesq ones in the late deceleration phase. The above findings on the basis of dimensional arguments are in accordance with our observation that the mixing for the gravity currents on the steeper unbounded uniform slopes in the late deceleration phase is more violent than the mixing for the gravity currents on the milder unbounded uniform slopes in the late deceleration phase.

Acknowledgements. A. D. is grateful for encouragement from Professors P. Linden and S. Dalziel at the University of Cambridge, S. Balachandar at the University of Florida, M. Garcia and G. Parker at the University of Illinois at Urbana-Champaign. Funding supports from Taiwan Ministry of Science and Technology through grant MOST-109-2628-E-002-006-MY2 and from National Taiwan University through grant 109L7830 are greatly acknowledged.

Declaration of interests. The authors report no conflict of interest.

Author ORCIDs.

(1) Albert Dai https://orcid.org/0000-0001-5139-8168.

\section{REFERENCES}

Adduce, C., Sciortino, G. \& Proietti, S. 2012 Gravity currents produced by lock-exchanges: experiments and simulations with a two layer shallow-water model with entrainment. J. Hydraul. Engng 138 (2), 111-121.

BAtChelor, G.K. 1967 An Introduction to Fluid Dynamics. Cambridge University Press.

BEghin, P., Hopfinger, E.J. \& BRITTER, R.E. 1981 Gravitational convection from instantaneous sources on inclined boundaries. J. Fluid Mech. 107, 407-422.

BonneCAZE, R.T. \& Lister, J.R. 1999 Particle-driven gravity currents down planar slopes. J. Fluid Mech. $390,75-91$.

Cantero, M., Balachandar, S. \& Garcia, M. 2007 High-resolution simulations of cylindrical density currents. J. Fluid Mech. 590, 437-469.

DAI, A. 2013a Experiments on gravity currents propagating on different bottom slopes. J. Fluid Mech. 731, $117-141$.

DAI, A. $2013 b$ Power-law for gravity currents on slopes in the decelerating phase. Dyn. Atmos. Oceans 63, 94-102.

DAI, A. 2014 Non-Boussinesq gravity currents propagating on different bottom slopes. J. Fluid Mech. 741, 658-680.

DAI, A. 2015 High-resolution simulations of downslope gravity currents in the acceleration phase. Phys. Fluids $27,076602$.

DAI, A. \& HUANG, Y.-L. 2016 High-resolution simulations of non-Boussinesq downslope gravity currents in the acceleration phase. Phys. Fluids 28, 026602.

DAI, A. \& HUANG, Y.-L. 2020 Experiments on gravity currents propagating on unbounded uniform slopes. Environ. Fluid Mech. 20, 1637-1662.

DAI, A. \& WU, C.-S. 2016 High-resolution simulations of cylindrical gravity currents in a rotating system. J. Fluid Mech. 806, 71-101.

Ellison, T.H. \& TURNER, J.S. 1959 Turbulent entrainment in stratified flows. J. Fluid Mech. 6, 423-448.

Hacker, J., Linden, P.F. \& DAlziel, S.B. 1996 Mixing in lock-release gravity currents. Dyn. Atmos. Oceans 24, 183-195.

Hallworth, M.A., Huppert, H.E., Phillips, J.C. \& Sparks, R.S.J. 1996 Entrainment into two-dimensional and axisymmetric turbulent gravity currents. J. Fluid Mech. 308, 289-311. 


\section{A. Dai and Y.-L. Huang}

Hopfinger, E.J. 1983 Snow avalanche motion and related phenomena. Annu. Rev. Fluid Mech. 15, 47-76.

Hoult, D.P. 1972 Oil spreading on the sea. Annu. Rev. Fluid Mech. 4, 341-368.

HuPPERT, H.E. \& SiMPSON, J. 1980 The slumping of gravity currents. J. Fluid Mech. 99, 785-799.

Inghilesi, R., Adduce, C., Lombardi, V., Roman, F. \& Armenio, V. 2018 Axisymmetric three-dimensional gravity currents generated by lock exchange. J. Fluid Mech. 851, 507-544.

Johnson, C.G. \& HoGG, A.J. 2013 Entraining gravity currents. J. Fluid Mech. 731, 477-508.

Jones, C.S., Cenedese, C., Chassignet, E.P., Linden, P.F. \& Sutherland, B.R. 2014 Gravity current propagation up a valley. J. Fluid Mech. 762, 417-434.

KorotKin, A.I. 2008 Added Masses of Ship Structures, 1st edn. Springer.

La Rocca, M., Adduce, C., Lombardi, V., Sciortino, G. \& Hinkermann, R. $2012 a$ Developement of a lattice Boltzmann method for two-layered shallow-water flow. Intl J. Numer. Meth. Fluids 70 (8), $1048-1072$.

La Rocca, M., Adduce, C., Sciortino, G., Bateman, P.A. \& Boniforti, M.A. $2012 b$ A two-layer shallow water model for 3D gravity currents. J. Hydraul. Res. 50 (2), 208-217.

La Rocca, M., Adduce, C., Sciortino, G. \& Pinzon, A.B. 2008 Experimental and numerical simulation of three-dimensional gravity currents on smooth and rough bottom. Phys. Fluids 20 (10), 106603.

Lombardi, V., Adduce, C., Sciortino, G. \& La Rocca, M. 2015 Gravity currents flowing upslope: laboratory experiments and shallow-water simulations. Phys. Fluids 27, 016602.

Marleau, L.J., Flynn, M.R. \& Sutherland, B.R. 2014 Gravity currents propagating up a slope. Phys. Fluids 26, 046605.

MAXWORTHY, T. 2010 Experiments on gravity currents propagating down slopes. Part 2. The evolution of a fixed volume of fluid released from closed locks into a long, open channel. J. Fluid Mech. 647, $27-51$.

MAXWORTHY, T. \& NOKES, R.I. 2007 Experiments on gravity currents propagating down slopes. Part 1. The release of a fixed volume of heavy fluid from an enclosed lock into an open channel. J. Fluid Mech. 584, 433-453.

Negretti, M.E., Flòr, J. -B. \& Hopfinger, E.J. 2017 Development of gravity currents on rapidly changing slopes. J. Fluid Mech. 833, 70-97.

Ottolenghi, L., Adduce, C., Inghilesi, R., Armenio, V. \& Roman, F. $2016 a$ Entrainment and mixing in unsteady gravity currents. J. Hydraul. Res. 54 (5), 541-557.

Ottolenghi, L., Adduce, C., Inghilesi, R., Roman, F. \& Armenio, V. $2016 b$ Mixing in lock-release gravity currents propagating up a slope. Phys. Fluids 28, 056604.

Ottolenghi, L., Adduce, C., Roman, F. \& ARmenio, V. 2017 a Analysis of the flow in gravity currents propagating up a slope. Ocean Model. 115, 1-13.

Ottolenghi, L., Cenedese, C. \& Adduce, C. $2017 b$ Entrainment in a dense current flowing down a rough sloping bottom in a rotating fluid. J. Phys. Oceanogr. 47 (3), 485-498.

Ouillon, R., Meiburg, E. \& Sutherland, B.R. 2019 Turbidity currents propagating down a slope into a stratified saline ambient fluid. Environ. Fluid Mech. 19 (5), 1143-1166.

PAWLAK, G. \& ARMI, L. 2000 Mixing and entrainment in developing stratified currents. J. Fluid Mech. 424, 45-73.

Rastello, M. \& Hopfinger, E.J. 2004 Sediment-entraining suspension clouds: a model of powder-snow avalanches. J. Fluid Mech. 509, 181-206.

Ross, A.N., LindEN, P.F. \& DALZIEL, S.B. 2002 A study of three-dimensional gravity currents on a uniform slope. J. Fluid Mech. 453, 239-261.

SAHURI, R.M., KAMinSKi, A.K., FlynN, M.R. \& Ungarish, M. 2015 Axisymmetric gravity currents in two-layer density-stratified media. Environ. Fluid Mech. 15, 1035-1051.

Shin, J., Dalziel, S. \& Linden, P.F. 2004 Gravity currents produced by lock exchange. J. Fluid Mech. 521, 1-34.

Simpson, J. 1997 Gravity Currents, 2nd edn. Cambridge University Press.

Steenhauer, K., Tokyay, T. \& Constantinescu, G. 2017 Dynamics and structure of planar gravity currents propagating down an inclined surface. Phys. Fluids 29 (3), 036604.

TiCKLE, G. 1996 A model of the motion and dilution of a heavy gas cloud released on a uniform slope in calm conditions. J. Hazard. Mater. 49, 29-47.

Ungarish, M. 2009 An Introduction to Gravity Currents and Intrusions. Chapman \& Hall/CRC Press.

Webber, D., Jones, S. \& MARTin, D. 1993 A model of the motion of a heavy gas cloud released on a uniform slope. J. Hazard. Mater. 33, 101-122.

Zgheib, N., OOI, A. \& Balachandar, S. 2016 Front dynamics and entrainment of finite circular gravity currents on an unbounded uniform slope. J. Fluid Mech. 801, 322-352. 